Communications in Physics, Vol. 29, No. 3 (2019), pp. 263-276

DOI:10.15625/0868-3166/29/3/13731

\title{
STUDY OF OXYGEN VACANCY DIFFUSION IN YTTRIA-DOPED CERIA AND YTTRIA-STABILIZED ZIRCONIA BY STATISTICAL MOMENT METHOD
}

\author{
LE THU LAM ${ }^{1, \dagger}$, VU VAN HUNG ${ }^{2}$ AND NGUYEN THANH HAI ${ }^{2}$ \\ ${ }^{1}$ Tay Bac University, Quyet Tam Precinct, Son La, Vietnam \\ ${ }^{2}$ University of Education, 182 Luong The Vinh Street, Thanh Xuan, Hanoi, Vietnam \\ ${ }^{3}$ The National Assembly of the Socialist Republic of Vietnam, \\ 22 Hung Vuong Street, Ba Dinh, Hanoi, Vietnam \\ ${ }^{\dagger}$ E-mail: lethulamtb@gmail.com
}

Received 4 April 2019

Accepted for publication 10 July 2019

Published 15 August 2019

\begin{abstract}
Oxygen vacancy diffusion in yttria-doped ceria (YDC) and yttria-stabilized zirconia (YSZ) are investigated using statistical moment method, including the anharmonicity effects of thermal lattice vibrations. The expressions of oxygen vacancy-dopant association energy and oxygen vacancy migration energy are derived in an explicit form. Calculation of the vacancy migration energy enables us to evaluate the important role of dopant cation on the oxygen vacancy diffusion. The dependences of the vacancy activation energies and diffusion coefficients in YDC and YSZ systems on dopant concentration are also discussed in detail. The calculated results are in good agreement with the other theoretical and experimental results.
\end{abstract}

Keywords: Oxygen vacancy diffusion; yttria-doped ceria; statistical moment method.

Classification numbers: 66.30.Lw; 68.43.Jk; 05.40.-a.

\section{INTRODUCTION}

Solid oxide fuel cells (SOFCs) are electrochemical devices that produce electricity directly from chemical energy. Nowadays, SOFCs have been widely used in automobile and power sources because of high efficiency, long operation life and low pollution [1]. Yttria-doped ceria (YDC) and yttria-stabilized zirconia (YSZ) with high ionic conductivity are the most popular materials used as the electrolytes for SOFCs operation in the intermediate temperature range. In the systems, (C)2019 Vietnam Academy of Science and Technology 
current is carried by oxygen vacancies that are generated to compensate for the lower charge of dopant cations $[2,3]$.

To understand the mechanism of oxygen vacancy transport in YDC and YSZ, a significant number of theoretical and experimental studies have been carried out. For YDC, H. Yoshida et al. [4] using extended X-ray absorption fine structure (EXAFS) measurements suggested that the oxygen vacancies tend to be trapped by the dopant cations. Fei Ye et al. [5] showed that the trapping effect arises from the formation of the defect cluster due to the associations between oxygen vacancies and dopant cations. These clusters can inhibit the hopping of oxygen vacancies and hence, decrease the diffusion coefficient of doped ceria. Further, the oxygen vacancy ordering in nano-sized domains with higher degree could block the vacancy transport more effectively [6]. The results obtained by first principle density function theory (DFT) calculations [7] revealed that determining the ionic conductivity in doped ceria is strongly affected by the lattice deformation. For YSZ, the diffusion coefficient of $\mathrm{O}^{2-}$ ions is apparently much larger than that of cations and decreases with an increase of the dopant concentration [8]. A study using ab initio and classical molecular dynamics (MD) simulations [9] suggested that the effect of vacancy-vacancy interaction could play an important role in determining the vacancy diffusion coefficient. More recently, A. Kushima et al. [10] showed the dependences of oxygen vacancy migration paths and edges on lattice strain. A decrease of migration edge arises from the increase of migration space and the weakening of vacancy-dopant associations. Remarkable, numerous theoretical studies $[11,13,14]$ have shown that, the presence of dopant ions in the common edge of two adjacent tetrahedra could limit available pathways for the oxygen vacancy diffusion in YDC and YSZ due to forming high-energy edges.

The diffusion coefficient of $\mathrm{CeO}_{2}$ with fluorite structure was investigated by statistical moment method (SMM) including the anharmonicity effects of thermal lattice vibrations [15]. The oxygen vacancies are thermally generated and the calculated vacancy activation energy equals three-eighth the interaction potential of an oxygen ion. In YDC and YSZ, the most oxygen vacancies are generated due to doping and therefore, the vacancy activation energies depend strongly on the dopant concentration. The present paper provides the explicitly analytic expression of the vacancy activation energy, taking into account the role of dopant cations using the SMM. The dependences of the vacancy activation energies and diffusion coefficients on the dopant concentration are discussed in detail. This study provides more insight into the atomistic level picture of the vacancy diffusion mechanism in solid oxide electrolyte materials.

\section{THEORY}

\section{II.1. Free energy}

Cubic $\mathrm{CeO}_{2}$ and $\mathrm{ZrO}_{2}$ have the fluorite crystal structure with eight cations $\left(\mathrm{Ce}^{4+}, \mathrm{Zr}^{4+}\right)$ occupying face-centered cubic $(f c c)$ lattice sites and four $\mathrm{O}^{2-}$ ions occupying cubic sublattice sites. Helmholtz free energy of $\mathrm{RO}_{2}$ system $(\mathrm{R}=\mathrm{Ce}, \mathrm{Zr})$ was written by taking into account the configuration entropies $\mathrm{S}_{c}$, via the Boltzmann relation as [16]

$$
\Psi_{R O_{2}}=C_{R} \Psi_{R}+C_{O} \Psi_{O}-T S_{c},
$$

where $C_{R}, C_{O}$ denote concentrations of $\mathrm{R}^{4+}, \mathrm{O}^{2-}$ ions, respectively, and $\Psi_{R}, \Psi_{O}$ are the Helmholtz free energies of $\mathrm{R}^{4+}, \mathrm{O}^{2-}$ ions, respectively. The configuration entropies $\mathrm{S}_{c}$ refer to the number of 
ways that ions pack together in the crystal lattice. In the harmonic approximation, $\Psi_{R}, \Psi_{O}$ have the forms

$$
\begin{aligned}
& \Psi_{R}=U_{0}^{R}+3 N_{R} \theta\left[x_{R}+\ln \left(1-e^{-2 x_{R}}\right)\right], \\
& \Psi_{O}=U_{0}^{O}+3 N_{O} \theta\left[x_{O}+\ln \left(1-e^{-2 x_{O}}\right)\right],
\end{aligned}
$$

with $U_{0}^{R}, U_{0}^{O}$ represent the sums of effective pair interaction energies for $\mathrm{R}^{4+}, \mathrm{O}^{2-}$ ions, respectively, and $x_{R}=\frac{\hbar \omega_{R}}{2 \theta}, x_{O}=\frac{\hbar \omega_{O}}{2 \theta}, \theta=k_{B} T$ ( $k_{B}$-the Boltzmann constant), and $\omega_{R}$ (or $\left.\omega_{O}\right)$ is the atomic vibration frequency of $\mathrm{R}^{4+}$ (or $\mathrm{O}^{2-}$ ) ions

$$
k_{R}=\frac{1}{2} \sum_{i}\left(\frac{\partial^{2} \varphi_{i 0}^{R}}{\partial u_{i \beta}^{2}}\right)_{e q}=m^{*} \omega_{R}^{2}, \quad k_{O}=\frac{1}{2} \sum_{i}\left(\frac{\partial^{2} \varphi_{i 0}^{O}}{\partial u_{i \beta}^{2}}\right)_{e q}=m^{*} \omega_{O}^{2},
$$

where $\beta=x, y$, or $z$, and $u_{i \beta}$ is $\beta$-Cartesian components of the displacement of $i$-th ion, $\varphi_{i 0}^{R}\left(\right.$ or $\left.\varphi_{i 0}^{O}\right)$ is the interaction potential between the 0 -th $\mathrm{R}^{4+}$ (or $\mathrm{O}^{2-}$ ) and the $i$-th ions, and $m^{*}$ is the average atomic mass of the system, $m^{*}=C_{R} m_{R}+C_{O} m_{O}$.

Doping $\mathrm{CeO}_{2}$ and $\mathrm{ZrO}_{2}$ with yttria $\left(\mathrm{Y}_{2} \mathrm{O}_{3}\right)$ replaces $\mathrm{R}^{4+}$ by $\mathrm{Y}^{3+}$ ions on the $f c c$ cation lattice and produces an oxygen vacancy for every two $\mathrm{Y}^{3+}$ ions to satisfy charge neutrality of the crystal lattice. If the yttrium concentration in YDC and YSZ systems is denoted by $x$ and there are $N$ cations in the crystal lattice, then the numbers of $\mathrm{R}^{4+}, \mathrm{Y}^{3+}, \mathrm{O}^{2-}$ ions and the oxygen vacancies in YDC and YSZ are $N_{R}=N(1-x), N_{Y}=N x, N_{O}=N(2-x / 2), N_{v a}=N x / 2$, respectively. Thus, the general chemical formula of YDC and YSZ systems can be written as $\mathrm{R}_{1-x} \mathrm{Y}_{x} \mathrm{O}_{2-x / 2}$.

The Helmholtz free energy of $\mathrm{R}_{1-x} \mathrm{Y}_{x} \mathrm{O}_{2-x / 2}$ system can be derived from the Helmholtz free energy of $\mathrm{RO}_{2-x / 2}$ system because of substituting $N_{Y} \mathrm{Y}^{3+}$ ions into the posititons of $\mathrm{R}^{4+}$ ions on the $f c c$ cation lattice of $\mathrm{RO}_{2-x / 2}$ system. Now, let us consider the simplest case that one $\mathrm{R}^{4+}$ ion is replaced by one $\mathrm{Y}^{3+}$ ion in $\mathrm{RO}_{2-x / 2}$ system. This substitution causes the change of the Gibbs free energy of the system as

$$
g_{v}^{f} \approx-u_{0}^{R}+\psi_{Y},
$$

with $u_{0}^{R}$ is the average interaction potential of a $\mathrm{R}^{4+}$ ion in $\mathrm{RO}_{2-x / 2}$ system, $\psi_{Y}$ is the Helmholtz free energy of a $\mathrm{Y}^{3+}$ ion in $\mathrm{R}_{1-x} \mathrm{Y}_{x} \mathrm{O}_{2-x / 2}$ system. Because $\mathrm{R}_{1-x} \mathrm{Y}_{x} \mathrm{O}_{2-x / 2}$ system is created by the substitution of $N_{Y} \mathrm{Y}^{3+}$ ions for $N_{Y} \mathrm{R}^{4+}$ ions in $\mathrm{RO}_{2-x / 2}$ system, then the Gibbs free energy of $\mathrm{R}_{1-x} \mathrm{Y}_{x} \mathrm{O}_{2-x / 2}$ system can be determined by the Gibbs free energy of $\mathrm{RO}_{2-x / 2}$ system, $G_{0}$,

$$
G=G_{0}+N_{Y} g_{v}^{f}-T S_{c}^{*}
$$

with $S_{c}^{*}$ is the configuration entropies of $\mathrm{R}_{1-x} \mathrm{Y}_{x} \mathrm{O}_{2-x / 2}$ system.

Substituting Eq. (5) into Eq. (6), one obtains the following formula

$$
G=G_{0}+N_{Y}\left(-u_{0}^{R}+\psi_{Y}\right)-T S_{c}^{*}=\Psi_{R O_{2-x / 2}}+N_{Y}\left(-u_{0}^{R}+\psi_{Y}\right)+P V-T S_{c}^{*},
$$

with $P$ is the hydrostatic pressure, $V$ is the volume of $\mathrm{R}_{1-x} \mathrm{Y}_{x} \mathrm{O}_{2-x / 2}$ system. From Eq. (7), the Helmholtz free energy of $\mathrm{R}_{1-x} \mathrm{Y}_{x} \mathrm{O}_{2-x / 2}$ system can be now derived

$$
\Psi=\Psi_{R O_{2-x / 2}}+N_{Y}\left(-u_{0}^{R}+\psi_{Y}\right)-T S_{c}^{*}=\Psi_{R O_{2-x / 2}}+\Psi_{Y}-N_{Y} u_{0}^{R}-T S_{c}^{*},
$$


here, $\Psi_{R O_{2-x / 2}}$ is determined by Eqs. (1) - (3) with $C_{R}=1 / 3, C_{O}=(2-x / 2) / 3, \Psi_{Y}$ is the total Helmholtz free energy of $\mathrm{Y}^{3+}$ ions. Because the sites of $f c c$ lattice are occupied by $\mathrm{Y}^{3+}$ ions, then the expression of $\Psi_{Y}$ is the same form as that of $\Psi_{R}$

$$
\Psi_{Y}=U_{0}^{Y}+3 N_{Y} \theta\left[x_{Y}+\ln \left(1-e^{-2 x_{Y}}\right)\right],
$$

with $U_{0}^{Y}$ is the total interaction potential of $\mathrm{Y}^{3+}$ ions in $\mathrm{R}_{1-x} \mathrm{Y}_{x} \mathrm{O}_{2-x / 2}$ system and $x_{Y}=\frac{\hbar \omega_{Y}}{2 \theta}$, with $\omega_{Y}$ is the vibration frequency of $\mathrm{Y}^{3+}$ ions in $\mathrm{R}_{1-x} \mathrm{Y}_{x} \mathrm{O}_{2-x / 2}$ system

$$
k_{Y}=\frac{1}{2} \sum_{i}\left(\frac{\partial^{2} \varphi_{i 0}^{Y}}{\partial u_{i \beta}^{2}}\right)_{e q}=m^{* *} \omega_{Y}^{2},
$$

with $m^{* *}$ is the average atomic mass of $\mathrm{R}_{1-x} \mathrm{Y}_{x} \mathrm{O}_{2-x / 2}$ system, $m^{* *}=C_{R} m_{R}+C_{Y} m_{Y}+C_{O} m_{O}$.

\section{II.2. The vacancy diffusion coefficient}

The vacancy diffusion coefficient of $\mathrm{CeO}_{2}$ was derived by V.V. Hung et al. [15]

$$
D=D_{0} \exp \left(-\frac{E_{a}}{k_{B} T}\right)
$$

where $E_{a}$ is the vacancy activation energy and the pre-exponential factor of the diffusion coefficient, $D_{0}$, is given as

$$
D_{0}=\frac{n_{1} f v r_{1}^{2} \exp \left(\frac{S_{v}^{f}}{k_{B}}\right)}{k_{B}},
$$

where $n_{1}$ is the number of $\mathrm{O}^{2-}$ ions at the first nearest neighbor positions with regard to the oxygen vacancy, the factor $f$ is correlation factor which represents the deviation from randomness of the ionic jumps, $v$ is the characteristic lattice frequency of $\mathrm{O}^{2-}$ ions, $r_{1}$ is the shortest distance between two lattice sites containing $\mathrm{O}^{2-}$ ions, $S_{v}^{f}$ is entropy for the formation of an oxygen vacancy. We will use this formula to calculate the diffusion coefficients of YDC and YSZ systems.

For pure $\mathrm{CeO}_{2}$ and $\mathrm{ZrO}_{2}$, the vacancy concentration is very low due to the high vacancy formation energy. The vacancy activation energy is the sum of the vacancy formation energy $E_{f}$ and the vacancy migration energy $E_{m}$. For YDC and YSZ, the oxygen vacancies and $\mathrm{Y}^{3+}$ ions are assumed as charged point defects with effective charges as +2 and -1 , respectively [17, 18]. Therefore, the bonds are created between the oxygen vacancies and $\mathrm{Y}^{3+}$ ions with vacancydopant association energy $E_{a s s}$ and prevent the migration of the oxygen vacancies. Hence, the number of the mobility oxygen vacancies is determined by the vacancy-dopant association energy. Consequently, the vacancy activation energy is determined as the sum of $E_{a s s}$ and $E_{m}$

$$
E_{a}=E_{a s s}+E_{m}
$$

In the next section, we will present the analytic expressions to calculate the vacancy-dopant association energy and the vacancy migration energy. 


\section{II.2.1. The vacancy-dopant association energy}

The associations between the oxygen vacancies and $\mathrm{Y}^{3+}$ ions form the charged defect clusters or electrically neutral clusters [19]. In these clusters, the oxygen vacancies tend to occupy either the first nearest neighbor sites $(1 \mathrm{NN})$ or the second nearest neighbor sites $(2 \mathrm{NN})$ to $\mathrm{Y}^{3+}$ ions.

The vacancy-dopant association energy in doped ceria and zirconia was calculated by atomistic simulation and/or MD methods [19,20]. The association energy between an oxygen vacancy $\mathrm{V}_{\mathrm{O}}^{\bullet \bullet}$ and $\mathrm{a} \mathrm{Y}^{3+}$ ion is the difference between the energy of the associated defect cluster $\mathrm{V}_{\mathrm{O}}^{\bullet \bullet}-\mathrm{Y}^{3+}$ and the energy sum of the isolated defects $\mathrm{V}_{\mathrm{O}}^{\bullet \bullet}$ and $\mathrm{Y}^{3+}$

$$
E_{\text {ass }}=\left[\Psi_{\text {total }}\left(\mathrm{V}_{\mathrm{O}}^{\bullet \bullet}-\mathrm{Y}^{3+}\right)\right]-\left[\Psi_{\text {total }}\left(\mathrm{V}_{\mathrm{O}}^{\bullet \bullet}\right)+\Psi_{\text {total }}\left(\mathrm{Y}^{3+}\right)\right] .
$$

To calculate the vacancy-dopant association energy using SMM, one considers the $\mathrm{R}_{N_{R}} \mathrm{Y}_{N_{Y}} \mathrm{O}_{N_{O}}$ system (called as the system I) with the Helmholtz free energy $\Psi_{\mathrm{R}_{N_{R}} \mathrm{Y}_{N_{Y}} \mathrm{O}_{N_{O}}}$, containing $N_{v a}$ Oxygen vacancies and $N_{Y} \mathrm{Y}^{3+}$ ions at the associated state. In these system, the replacing a $\mathrm{R}^{4+}$ by a $\mathrm{Y}^{3+}$ ion will create the $\mathrm{R}_{N_{R}-1} \mathrm{Y}_{N_{Y}+1} \mathrm{O}_{N_{O}}$ system (called as the system II) with the Helmholtz free energy $\Psi_{\mathrm{R}_{N_{R}-1} \mathrm{Y}_{N_{Y}+1} \mathrm{O}_{N_{O}}}$. The system II also has the associations between $N_{Y} \mathrm{Y}^{3+}$ ions and $N_{v a}$ oxygen vacancies but unlike the system $\mathrm{I}$, this system has more an $\mathrm{Y}^{3+}$ ion at the isolated state. It is noted that each oxygen vacancy is associated with two $\mathrm{Y}^{3+}$ ions because the substitution of $\mathrm{Y}^{3+}$ ions for $\mathrm{R}^{4+}$ is accompanied by the formation of an oxygen vacancy for every two $\mathrm{Y}^{3+}$ ions. For this reason, $\mathrm{R}_{N_{R}-1} \mathrm{Y}_{N_{Y}+1} \mathrm{O}_{N_{O}-1}$ system (called as the system III) with the Helmholtz free energy $\Psi_{\mathrm{R}_{N_{R}-1} \mathrm{Y}_{N_{Y}+1} \mathrm{O}_{N_{O^{-1}}}}$ has an isolated oxygen vacancy. By adding a $\mathrm{Y}^{3+}$ ion to the system III, the $\mathrm{R}_{N_{R}-2} \mathrm{Y}_{N_{Y}+2} \mathrm{O}_{N_{O}-1}$ system (called as the system IV) is formed with the Helmholtz free energy $\Psi_{\mathrm{R}_{N_{R}-2} \mathrm{Y}_{N_{Y}+2} \mathrm{O}_{N_{O^{-1}}}}$. In this system, $\left(N_{v a}+1\right)$ oxygen vacancies are associated with $\left(N_{Y}+2\right) \mathrm{Y}^{3+}$ ions. Based on Eq. (14), the vacancy-dopant association energy is determined as the Helmholtz free energy difference between the systems containing the oxygen vacancies and $\mathrm{Y}^{3+}$ ions at the associated state (the systems I and IV) and the systems containing the oxygen vacancies and $\mathrm{Y}^{3+}$ ions at the isolated state (the systems II and III)

$$
E_{\text {ass }}=\left(\Psi_{\mathrm{R}_{N_{R}} \mathrm{Y}_{N_{Y}} \mathrm{O}_{N_{O}}}+\Psi_{\mathrm{R}_{N_{R}-2} \mathrm{Y}_{N_{Y}+2} \mathrm{O}_{N^{-1}}}\right)-\left(\Psi_{\mathrm{R}_{N_{R}-1} \mathrm{Y}_{N_{Y}+1} \mathrm{O}_{N_{O}}}+\Psi_{\mathrm{R}_{N_{R}-1} \mathrm{Y}_{N_{Y}+1} \mathrm{O}_{N_{O^{-1}}}}\right),
$$

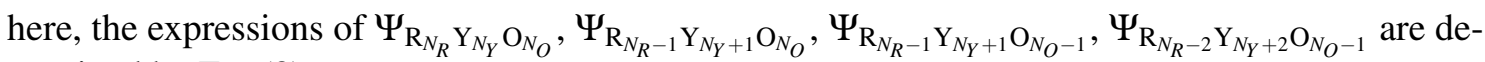
termined by Eq. (8).

\section{II.2.2. The vacancy migration energy}

In YDC and YSZ systems, the oxygen vacancies hop dominantly along the $<100>$ direction from the lattice sites $[11,13]$. The movement of an oxygen vacancy between the adjacent sites in the crystal lattice corresponds to the migration of an opposite oxygen ion in the reverse direction. Fig. 1 presents the migration of an $\mathrm{O}^{2-}$ ion from the lattice site $\mathrm{A}$, across the saddle point $\mathrm{B}$ and occupying a vacant site $\mathrm{C}$. The states of crystal lattice before the oxygen migration from the site A and after the oxygen diffusion to the saddle point B are called as the initial state and the saddle point state, respectively. Thus, the energy for the vacancy migration is determined by

$$
E_{m}=\Psi_{0}-\Psi_{\text {saddle }}
$$



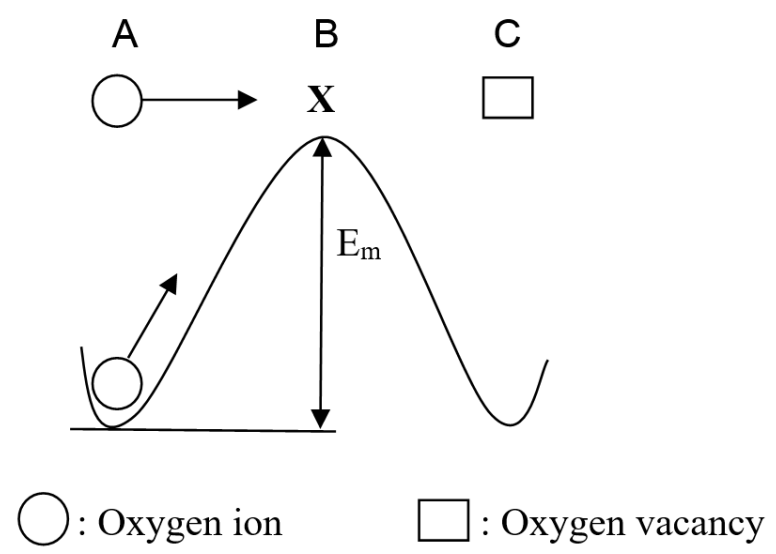

Fig. 1. An $\mathrm{O}^{2-}$ ion hops from the lattice site $\mathrm{A}$, across the saddle point $\mathrm{B}$ and occupies an adjacent vacant site $\mathrm{C}$.

with $\Psi_{0}$ is the free energy of system at the initial state and $\Psi_{\text {saddle }}$ is the free energy of system at the saddle point state. These free energies could be obtained from Eq. (8) with Eqs. (2), (3), (9) determining the total free energies of $\mathrm{R}^{4+}, \mathrm{O}^{2-}, \mathrm{Y}^{3+}$ ions. It is required to calculate the total interaction potentials $U_{0}^{R}, U_{0}^{O}$ and $U_{0}^{Y}$ of $\mathrm{R}^{4+}, \mathrm{O}^{2-}$, and $\mathrm{Y}^{3+}$ ions, respectively, at the initial and the saddle-point states.

a. The total interaction potentials of $\mathrm{R}^{4+}, \mathrm{O}^{2-}$, and $\mathrm{Y}^{3+}$ ions at the initial state

Firstly, we find the expression determining the total interaction potential of $\mathrm{O}^{2-}$ ions in $\mathrm{RO}_{2-x / 2}$ system. Due to the presence of the oxygen vacancies in the crystal lattice, the interaction potentials of $\mathrm{O}^{2-}$ ions are not similar. It is required to determine the average potential energy of an $\mathrm{O}^{2-}$ ion, $u_{O}$. The expression of $u_{O}$ is determined by the interactions between an $\mathrm{O}^{2-}$ ion and the surrounding ions

$$
u_{O}=u_{O-O}+u_{O-R}
$$

with $u_{O-O}, u_{O-R}$ are the average interaction potentials between an $\mathrm{O}^{2-}$ ion and surrounding $\mathrm{O}^{2-}$ and $\mathrm{R}^{4+}$ ions, respectively.

In order to determine $u_{O-O}$, one considers the $i$-th nearest-neighbor sites relative to a certain oxygen vacancy, $\mathrm{V}_{\mathrm{O}}^{\bullet \bullet}$. The number of $i$-th nearest-neighbor sites occupied by $\mathrm{O}^{2-}$ ions is $b_{i}^{O-O}$. In the crystal lattice, there are $N_{O} \mathrm{O}^{2-}$ ions and these ions could occupy $(2 N-1)$ the remaining sites. Therefore, probability that a lattice site is occupied by an $\mathrm{O}^{2-}$ ion as

$$
W_{O-O}=\frac{N_{O}}{2 N-1} .
$$

The number of $\mathrm{O}^{2-}$ ions occupied the $i$-th nearest-neighbor sites of $\mathrm{V}_{\mathrm{O}}^{\bullet \bullet}$ can be given by

$$
c_{i}^{O-O}=b_{i}^{O-O} W_{O-O},
$$

and $c_{i}^{O-O}$ is also the number of $\mathrm{O}^{2-}$ ions that have $\mathrm{V}_{\mathrm{O}}^{\bullet \bullet}$ at the $i$-th nearest-neighbor sites. Because the crystal lattice has $N_{v a}$ oxygen vacancies, then there are $N_{v a} c_{i}^{O-O} \mathrm{O}^{2-}$ ions having $\mathrm{V}_{\mathrm{O}}^{\bullet \bullet}$ ion at 
$i$-th nearest-neighbor sites. Subsequently, the number of the associations of $N_{O} \mathrm{O}^{2-}$ ions with surrounding $\mathrm{O}^{2-}$ ions at the $i$-th nearest-neighbor sites can be written as

$$
N_{i}^{O-O}=N_{O} b_{i}^{O-O}-N_{v a} c_{i}^{O-O} .
$$

From Eq. (20), we derive the average number of the associations of an $\mathrm{O}^{2-}$ ions with other $\mathrm{O}^{2-}$ ions at the $i$-th nearest-neighbour sites

$$
n_{i}^{O-O}=\frac{N_{i}^{O-O}}{N_{O}}=b_{i}^{O-O}\left(1-\frac{N_{v a}}{2 N-1}\right),
$$

therefore, the expression of $u_{O-O}$ is given by

$$
u_{O-O}=\left(1-\frac{N_{v a}}{2 N-1}\right) \sum_{i} b_{i}^{O-O} \varphi_{i 0}^{* O-O},
$$

with $\varphi_{i 0}^{* O-O}$ is the interaction potential between the 0 -th $\mathrm{O}^{2-}$ ion and an ion $\mathrm{O}^{2-}$ at the $i$-th nearestneighbor sites relative to the 0 -th $\mathrm{O}^{2-}$ ion.

In the same way, we obtain the expressions of $u_{O-R}$ and derive the formula of the total interaction potential of $\mathrm{O}^{2-}$ ions in $\mathrm{RO}_{2-x / 2}$ system

$$
U_{0}^{O}=\frac{N_{O}}{2}\left(\sum_{i} b_{i}^{O-R} \varphi_{i 0}^{* O-R}+\left(1-\frac{N_{v a}}{2 N-1}\right) \sum_{i} b_{i}^{O-O} \varphi_{i 0}^{* O-O}\right)
$$

Similar to the way of calculation for $U_{0}^{O}$, we have the expressions of the total interaction potential of $\mathrm{R}^{4+}$ ions in $\mathrm{RO}_{2-x / 2}$ system and the total interaction potential of $\mathrm{Y}^{3+}$ ions in $\mathrm{R}_{1-x} \mathrm{Y}_{x} \mathrm{O}_{2-x / 2}$ system

$$
\begin{gathered}
U_{0}^{R}=\frac{N_{R}}{2}\left(\sum_{i} b_{i}^{R-R} \varphi_{i 0}^{* R-R}+\left(1-\frac{N_{v a}}{2 N}\right) \sum_{i} b_{i}^{R-O} \varphi_{i 0}^{* R-O}\right) \\
U_{0}^{Y}=\frac{N_{Y}}{2}\left(\frac{N_{R}}{N-1} \sum_{i} b_{i}^{Y-R} \varphi_{i 0}^{* Y-R}+\frac{N_{Y}-1}{N-1} \sum_{i} b_{i}^{Y-Y} \varphi_{i 0}^{* Y-Y}+\left(1-\frac{N_{v a}}{2 N}\right) \sum_{i} b_{i}^{Y-O} \varphi_{i 0}^{* Y-O}\right),
\end{gathered}
$$

where $b_{i}^{X-O}$ (or $b_{i}^{X-R}$, or $b_{i}^{X-Y}$ ) is the number of the $i$-th nearest-neighbor sites relative to $\mathrm{X}$ ion (X $=\mathrm{O}^{2-}, \mathrm{R}^{4+}, \mathrm{Y}^{3+}$ ) that $\mathrm{O}^{2-}\left(\right.$ or $\mathrm{R}^{4+}$, or $\mathrm{Y}^{3+}$ ) ions could occupy, respectively, $\varphi_{i 0}^{* X-O}$ (or $\varphi_{i 0}^{* X-R}$, or $\varphi_{i 0}^{* X-Y}$ ) is the interaction potential between the 0 -th $\mathrm{X}$ ion and an ion $\mathrm{O}^{2-}\left(\right.$ or $\mathrm{R}^{4+}$, or $\mathrm{Y}^{3+}$ ) at the $i$-th nearest-neighbor sites relative to this $\mathrm{X}$ ion, respectively.

b. The total interaction potentials of $\mathrm{R}^{4+}, \mathrm{O}^{2-}$, and $\mathrm{Y}^{3+}$ ions at the saddle-point state

Firstly, the average interaction potential of an $\mathrm{O}^{2-}$ ion at the saddle-point state is given by

$$
u_{O}^{B}=u_{O}+\Delta u_{O}^{O-O}+\Delta u_{O}^{*},
$$

where $u_{O}$ is the average interaction potential of an $\mathrm{O}^{2-}$ at initial state, $\Delta u_{O}^{O-O}$ is the change in the average interaction potential of an $\mathrm{O}^{2-}$ ion arising from the interaction with the surrounding $\mathrm{O}^{2-}$ ions (see Appendix), and $\Delta u_{O}^{*}$ is the change in the average interaction potential of an $\mathrm{O}^{2-}$ 
ion arising from the interaction with the surrounding $\mathrm{R}^{4+}$ ions (denoted as $\Delta u_{O}^{O-R}$ ) and $\mathrm{Y}^{3+}$ ions (denoted as $\Delta u_{O}^{O-Y}$ )

$$
\Delta u_{O}^{*}=\Delta u_{O}^{O-R}+\Delta u_{O}^{O-Y} .
$$

Because of the oxygen movement, the interaction potentials between the diffusing oxygen ion at the site $\mathrm{A}$ and surrounding $\mathrm{R}^{4+}$ and $\mathrm{Y}^{3+}$ cations are lost and more the interaction potentials between the diffusing oxygen ion at the point $\mathrm{B}$ and these cations. Therefore, $\Delta u_{O}^{O-R}$ and $\Delta u_{O}^{O-Y}$ could be determined as

$$
\Delta u_{O}^{O-R}=\frac{\varphi_{O-R}^{B}-\varphi_{O-R}^{A}}{N_{O}}, \quad \Delta u_{O}^{O-Y}=\frac{\varphi_{O-Y}^{B}-\varphi_{O-Y}^{A}}{N_{O}},
$$

with $\varphi_{O-R}^{A, B}$ (or $\varphi_{O-Y}^{A, B}$ ) are the interaction potentials between the diffusing oxygen ion $\mathrm{O}^{2-}$ and surrounding $\mathrm{R}^{4+}$ (or $\mathrm{Y}^{3+}$ ) ions at the sites $\mathrm{A}$ and $\mathrm{B}$, respectively. The interaction potentials $\varphi_{O-R}^{A, B}$, $\varphi_{O-Y}^{A, B}$ depend sensitively on the configurations of the neighboring cations around the diffusing vacancy-oxygen ion pair. There are three main configurations of $\mathrm{R}^{4+}$ and $\mathrm{Y}^{3+}$ ions at the first neighbor sites around this diffusing pair (Fig. 2). These configurations generate three cation edges, namely, $\mathrm{R}^{4+}-\mathrm{R}^{4+}, \mathrm{R}^{4+}-\mathrm{Y}^{3+}$ and $\mathrm{Y}^{3+}-\mathrm{Y}^{3+}$.

0

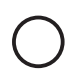

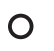

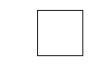

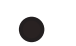

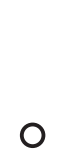

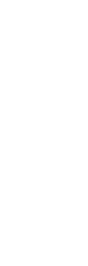

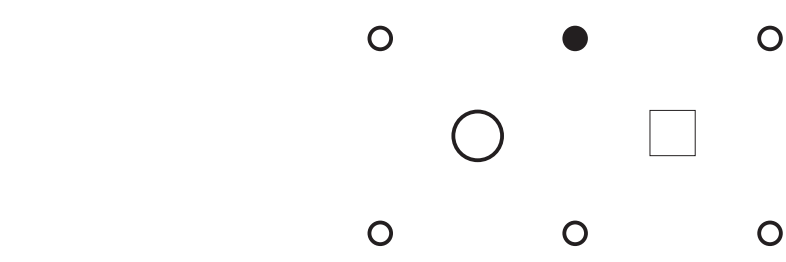

a.The $\mathrm{R}^{4+}-\mathrm{R}^{4+}$ edge

b. The $\mathrm{R}^{4+}-\mathrm{Y}^{3+}$ edge

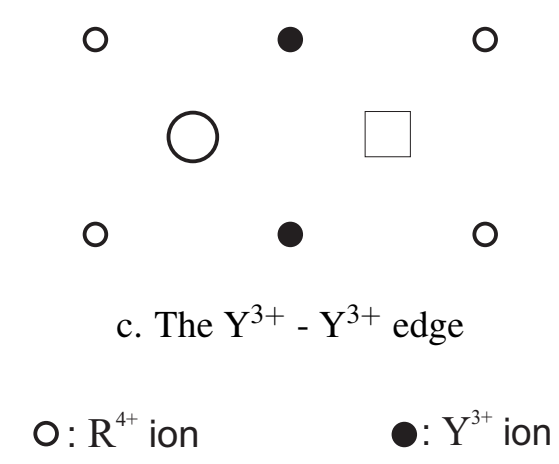

Fig. 2. Three configurations of neighboring cations around the diffusing vacancy-oxygen ion pair with three cation edges in YDC and YSZ system in two-dimension plane.

The average interaction potentials of a $\mathrm{R}^{4+}$ ion and a $\mathrm{Y}^{3+}$ ion at the saddle-point state are determined as

$$
u_{R}^{B}=u_{R}+\Delta u_{R}^{*}, \quad u_{R}^{Y}=u_{Y}+\Delta u_{Y}^{*},
$$

where $u_{R}$ and $u_{Y}$ are the average interaction potentials of a $\mathrm{R}^{4+}$ ion and a $\mathrm{Y}^{3+}$ ion at the initial state, respectively, and $\Delta u_{R}^{*}, \Delta u_{Y}^{*}$ are the changes in the average interaction potentials of a $\mathrm{R}^{4+}$ ion 
and a $\mathrm{Y}^{3+}$ ion due to the oxygen migration. The expressions of $\Delta u_{R}^{*}$ and $\Delta u_{Y}^{*}$ could be determined by $\varphi_{O-R}^{A, B}$ and $\varphi_{O-Y}^{A, B}$, respectively,

$$
\Delta u_{R}^{*}=\frac{\varphi_{O-R}^{B}-\varphi_{O-R}^{A}}{N_{R}}, \quad \Delta u_{Y}^{*}=\frac{\varphi_{O-Y}^{B}-\varphi_{O-Y}^{A}}{N_{Y}} .
$$

From Eqs. (26) - (30), we obtain the expressions of the total interaction potentials of $\mathrm{O}^{2-}$, $\mathrm{R}^{4+}, \mathrm{Y}^{3+}$ ions at the saddle-point state

$$
\begin{gathered}
U_{\text {saddle }}^{O}=U_{0}^{O}+\frac{\varphi_{O-R}^{B}+\varphi_{O-Y}^{B}-\varphi_{O-R}^{A}-\varphi_{O-Y}^{A}}{2}+\frac{N_{O} \Delta u_{O}^{O-O}}{2} \\
U_{\text {saddle }}^{R}=U_{0}^{R}+\frac{\varphi_{O-R}^{B}-\varphi_{O-R}^{A},}{2}, \quad U_{\text {saddle }}^{Y}=U_{0}^{Y}+\frac{\varphi_{O-Y}^{B}-\varphi_{O-Y}^{A}}{2} .
\end{gathered}
$$

\section{RESULTS AND DISCUSSION}

The interactions between ions in YDC and YSZ systems with fluorite structure including the long-range Coulomb interaction and the short-range interactions are described by a simple two-body potential of the Buckingham form $[19,21]$

$$
\varphi_{i j}(r)=\frac{q_{i} q_{j}}{r}+A_{i j} \exp \left(-\frac{r}{B_{i j}}\right)-\frac{C_{i j}}{r^{6}},
$$

where $q_{i}$ and $q_{j}$ are the charges of ion $i$ and $j$, respectively, $r$ is the distance between them and $A_{i j}, B_{i j}$ and $C_{i j}$ are the empirical parameters (listed in Table 1). The first term could be summed explicitly by using the Wolf method to turn the Coulomb interaction effectively into spherically symmetric potentials with relatively short-ranges [22]

$$
u_{i j}(r)=q_{i} q_{j}\left\{\frac{\operatorname{erfc}(\alpha r)}{r}-\frac{\operatorname{erfc}\left(\alpha R_{c}\right)}{R_{c}}+\left[\frac{\operatorname{erfc}\left(\alpha R_{c}\right)}{R_{c}^{2}}+\frac{2 \alpha \operatorname{erfc}\left(-\alpha^{2} R_{c}^{2}\right)}{\pi^{\frac{1}{2}}}\right]\left(r-R_{c}\right)\right\}, r \leq R_{c}
$$

where $\alpha$ is the damping parameter and $R_{c}$ is the cutoff radius. Based on report by P. Demontis $e t$ al. [22], the optimum values of $\alpha$ and $R_{c}$ are found for YDC and YSZ as $\alpha_{\mathrm{YDC}}=0.31 \AA^{-1}, R_{c}^{\mathrm{YDC}}$ $=11.715 \AA$ and $\alpha_{\mathrm{YSZ}}=0.34 \AA^{-1}, R_{c}^{\mathrm{YSZ}}=10.911 \AA$, respectively. The values of the cutoff radius allow us to limit the crystal lattice region for calculations. This region consists of 256 lattice sites for cations and 512 lattice sites for $\mathrm{O}^{2-}$ ions and oxygen vacancies.

Table 1. The parameters of the Buckingham potential in YDC and YSZ systems.

\begin{tabular}{|c|c|c|c|c|}
\hline Material & Interaction & $A_{i j} / \mathrm{eV}$ & $B_{i j} / \AA$ & $C_{i j} / \mathrm{eV}^{\circ} \AA^{6}$ \\
\hline \multirow{3}{*}{$\mathrm{YDC}[23]$} & $\mathrm{O}^{2-}-\mathrm{O}^{2-}$ & 9547.96 & 0.2192 & 32 \\
\cline { 2 - 5 } & $\mathrm{Ce}^{4+}-\mathrm{O}^{2-}$ & 1809.68 & 0.3547 & 20.40 \\
\cline { 2 - 5 } & $\mathrm{Y}^{3+}-\mathrm{O}^{2-}$ & 1766.4 & 0.3385 & 19.43 \\
\hline \multirow{3}{*}{$\mathrm{YSZ}[24]$} & $\mathrm{O}^{2-}-\mathrm{O}^{2-}$ & 9547.96 & 0.224 & 32 \\
\cline { 2 - 5 } & $\mathrm{Zr}^{4+}-\mathrm{O}^{2-}$ & 1502.11 & 0.345 & 5.1 \\
\cline { 2 - 5 } & $\mathrm{Y}^{3+}-\mathrm{O}^{2-}$ & 1366.35 & 0.348 & 19.6 \\
\hline
\end{tabular}


Based on the minimum condition of the potential energy of the systems at $T=0 \mathrm{~K}$, Eqs. (23) - (25) and (31) - (32) enable us to calculate the lattice constants at the initial and saddle point states, respectively. The lattice constants at these states in the dopant concentration range of $0.1-0.4$ are presented in Table 2 for YDC and Table 3 for YSZ. At the saddle point state, we evaluate the lattice constants at three situations corresponding to the oxygen movement across three cation edges, $\mathrm{R}^{4+}-\mathrm{R}^{4+}, \mathrm{R}^{4+}-\mathrm{Y}^{3+}$ and $\mathrm{Y}^{3+}-\mathrm{Y}^{3+}$. One can see that the crystal lattice is slightly deformed by the vacancy hopping in the overall dopant concentration. For YDC and YSZ, the values of lattice constants at the saddle point state $a_{\text {saddle }}$ for the $\mathrm{R}^{4+}-\mathrm{R}^{4+}, \mathrm{R}^{4+}-\mathrm{Y}^{3+}$ cation edges are larger than those at the initial state $a_{\text {initial }}$. However, the values of $a_{\text {saddle }}$ for the $\mathrm{Y}^{3+}$ $\mathrm{Y}^{3+}$ cation edge are smaller than those of $a_{\text {initial }}$. The larger space for the vacancy migration could promote the diffusion process and vice versa [13]. Therefore, we predict that the oxygen vacancies can migrate across the $\mathrm{R}^{4+}-\mathrm{R}^{4+}, \mathrm{R}^{4+}-\mathrm{Y}^{3+}$ cation edges while this transport is inhibited by the $\mathrm{Y}^{3+}-\mathrm{Y}^{3+}$ cation edge.

Table 2. The lattice constants at $0 \mathrm{~K}$ of YDC at the initial and saddle point states.

\begin{tabular}{|c|c|c|c|c|c|c|}
\hline \multicolumn{2}{|c|}{$x$} & 0.1 & 0.2 & 0.3 & 0.4 & 0.5 \\
\hline \multicolumn{2}{|c|}{$a_{\text {initial }} / \AA$} & 5.4091 & 5.4068 & 5.4044 & 5.4019 & 5.3993 \\
\hline \multirow{3}{*}{$a_{\text {saddle }} / \AA$} & $\mathrm{Ce}^{4+}-\mathrm{Ce}^{4+}$ & 5.4098 & 5.4075 & 5.4052 & 5.4027 & 5.4002 \\
\cline { 2 - 7 } & $\mathrm{Ce}^{4+}-\mathrm{Y}^{3+}$ & 5.4095 & 5.4073 & 5.4049 & 5.4025 & 5.3999 \\
\cline { 2 - 7 } & $\mathrm{Y}^{3+}-\mathrm{Y}^{3+}$ & 5.4085 & 5.4063 & 5.4040 & 5.4017 & 5.3991 \\
\hline
\end{tabular}

Table 3. The lattice constants at $0 \mathrm{~K}$ of YSZ at the initial and saddle point states.

\begin{tabular}{|c|c|c|c|c|c|c|}
\hline \multicolumn{2}{|c|}{$x$} & 0.1 & 0.2 & 0.3 & 0.4 & 0.5 \\
\hline \multicolumn{2}{|c|}{$a_{\text {initial }} / \AA$} & 5.1005 & 5.1172 & 5.1347 & 5.1530 & 5.1721 \\
\hline \multirow{3}{*}{$a_{\text {saddle }} / \AA$} & $\mathrm{Zr}^{4+}-\mathrm{Zr}^{4+}$ & 5.1010 & 5.1178 & 5.1354 & 5.1537 & 5.1729 \\
\cline { 2 - 7 } & $\mathrm{Zr}^{4+}-\mathrm{Y}^{3+}$ & 5.1008 & 5.1176 & 5.1351 & 5.1534 & 5.1726 \\
\cline { 2 - 7 } & $\mathrm{Y}^{3+}-\mathrm{Y}^{3+}$ & 5.1001 & 5.1169 & 5.1344 & 5.1527 & 5.1718 \\
\hline
\end{tabular}

To evaluate exactly the effect of dopant cations on the vacancy diffusion, it is required to calculate the energies for oxygen vacancy migration across three cation edges, $\mathrm{R}^{4+}-\mathrm{R}^{4+}, \mathrm{R}^{4+}$ $\mathrm{Y}^{3+}, \mathrm{Y}^{3+}-\mathrm{Y}^{3+}$. Using the different expressions of $\varphi_{O-R}^{A, B}$ and $\varphi_{O-Y}^{A, B}$ in Eqs. (31), (32) for three neighbour cation configurations in Fig. 2, we can determine the vacancy migration energies across the cation edges. The obtained results are presented in Table 4. It is clearly seen that the vacancy migration energies are sensitive to the cation edges. The migration energies have the smallest values without any dopant in the cation edges, and they increase with the presence of dopant in the edges. With the largest migration energy values, almost oxygen movement don't take place across the $\mathrm{Y}^{3+}-\mathrm{Y}^{3+}$ edge. Therefore, we can conclude that the presence of host cation in the cation edge promotes the vacancy hopping, while that of dopant cation in the cation edge blocks this movement. The effect of dopant cation on the vacancy diffusion could arise from two main 
factors. First, the oxygen vacancies move in the smaller space due to the bigger ionic radius of dopant cation compared with host cations. Second, the association between defects could trap the oxygen vacancies and prevents them from being mobile. The calculated results using the DFT method [11-14] have also been reported to confirm the role of dopant cation on the vacancy migration.

Table 4. The vacancy migration energies across the $\mathrm{R}^{4+}-\mathrm{R}^{4+}, \mathrm{R}^{4+}-\mathrm{Y}^{3+}, \mathrm{Y}^{3+}-\mathrm{Y}^{3+}$ cation edges in YDC and YSZ systems.

\begin{tabular}{|c|c|c|c|c|}
\hline \multirow{2}{*}{$E_{m}$} & Method & $\mathrm{R}^{4+}-\mathrm{R}^{4+}$ & $\mathrm{R}^{4+}-\mathrm{Y}^{3+}$ & $\mathrm{Y}^{3+}-\mathrm{Y}^{3+}$ \\
\hline \multirow{3}{*}{ YDC } & SMM & 0.2334 & 0.7295 & 1.0521 \\
\cline { 2 - 5 } & \multirow{2}{*}{$\mathrm{DFT}$} & $0.48[11]$ & $0.533[11]$ & $0.8[11]$ \\
\cline { 3 - 5 } & & $0.52[12]$ & $0.57[12]$ & $0.82[12]$ \\
\hline \multirow{3}{*}{ YSZ } & SMM & 0.3625 & 1.0528 & 1.5091 \\
\cline { 2 - 5 } & \multirow{2}{*}{$\mathrm{DFT}$} & $0.2[14]$ & $1.19[14]$ & $1.23[14]$ \\
\cline { 3 - 5 } & & $0.58[13]$ & $1.29[13]$ & $1.86[13]$ \\
\hline
\end{tabular}
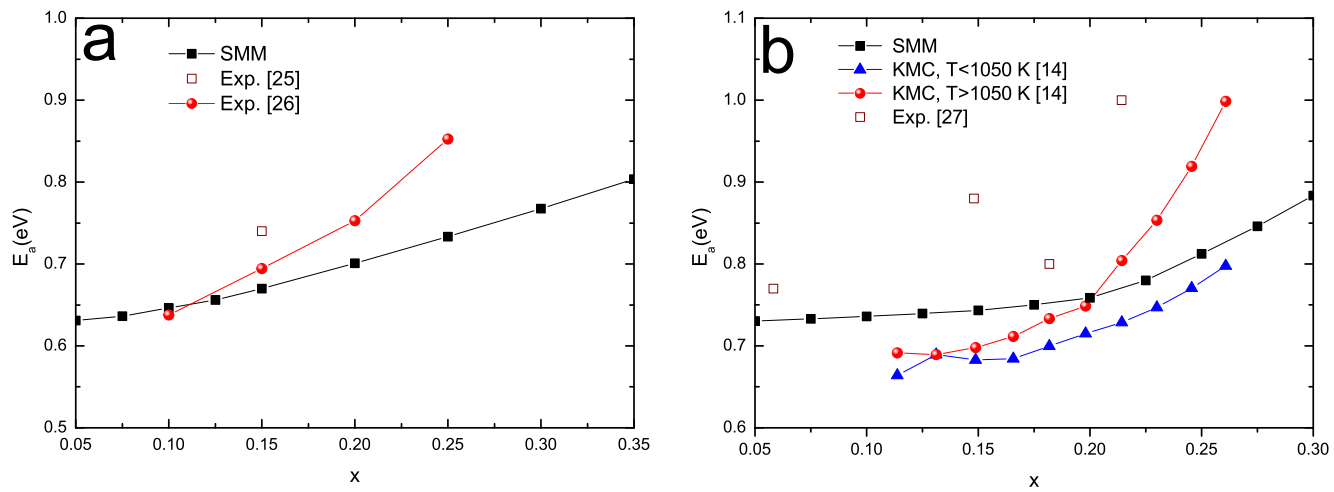

Fig. 3. The dopant concentration dependence of the calculated activation energies of YDC at $T=773 \mathrm{~K}$ (a) and YSZ at $T=1000 \mathrm{~K}$ (b). The theoretical results using kinetic Monte Carlo (KMC) simulation at $T>1050 \mathrm{~K}$ and $T<1050 \mathrm{~K}$ [14], and the experimental results around $773 \mathrm{~K}$ for YDC [25,26] and $1000 \mathrm{~K}$ for YSZ [27] are also displayed for comparison.

In Fig. 3, the SMM values of the vacancy activation energies are plotted as a function of the dopant concentration in YDC at $773 \mathrm{~K}$ and YSZ at $1000 \mathrm{~K}$. It is noted that the relation between the yttria concentration $y$ and the yttrium concentration $x$ in the systems is $y=x /(2-x)$ [13]. At low dopant concentration, the number of high energy edges $\mathrm{R}^{4+}-\mathrm{Y}^{3+}, \mathrm{Y}^{3+}-\mathrm{Y}^{3+}$ is small and the 
activation energies are nearly equal those for the vacancy migration across the $\mathrm{R}^{4+}-\mathrm{R}^{4+}$ edge. As dopant concentration increases, the oxygen-vacancy exchange through the $\mathrm{R}^{4+}-\mathrm{Y}^{3+}, \mathrm{Y}^{3+}-\mathrm{Y}^{3+}$ edges rather than $\mathrm{R}^{4+}-\mathrm{R}^{4+}$ edge can be expected to increase. Therefore, it is clearly seen that the calculated activation energies show an upward trend with the increasing of $x$ from 0 to 0.35 . The simulation and experimental results for the activation energies in YDC around $773 \mathrm{~K}$ [25,26] and YSZ around $1000 \mathrm{~K}[14,27]$ are also presented in Fig. 3. These results show almost the same tendency with the SMM results. However, the values of SMM results are slightly smaller than those of the experimental results. This is probably caused by neglecting the role of the vacancyvacancy interaction. Moreover, the vacancy migration along grain boundaries in polycrystalline samples could block the vacancy migration and increases the activation energies.

Fig. 4 presents Arrhenius plots of $D$ vs. $\left(1 / k_{B} T\right)$ (Eq. 11) at the different dopant concentrations for the vacancy diffusion in YDC and YSZ. The diffusion coefficients increase with the increasing temperature. The larger values of diffusion coefficients at the smaller dopant concentrations show that the diffusion coefficients decrease with an increase of dopant concentration. The MD [8,21] and experimental [28,29] results also show almost the same tendency. The dopant concentration dependence of the diffusion coefficients arises from the effect of dopant cations in the cation edges on the oxygen-vacancy exchange. At the high dopant concentration, an increased number of high energy edges generates the large activation energies and therefore, reduces the vacancy diffusion coefficients. One can see that the SMM values of vacancy diffusion coefficients are consistent with the experimental data [28,29].
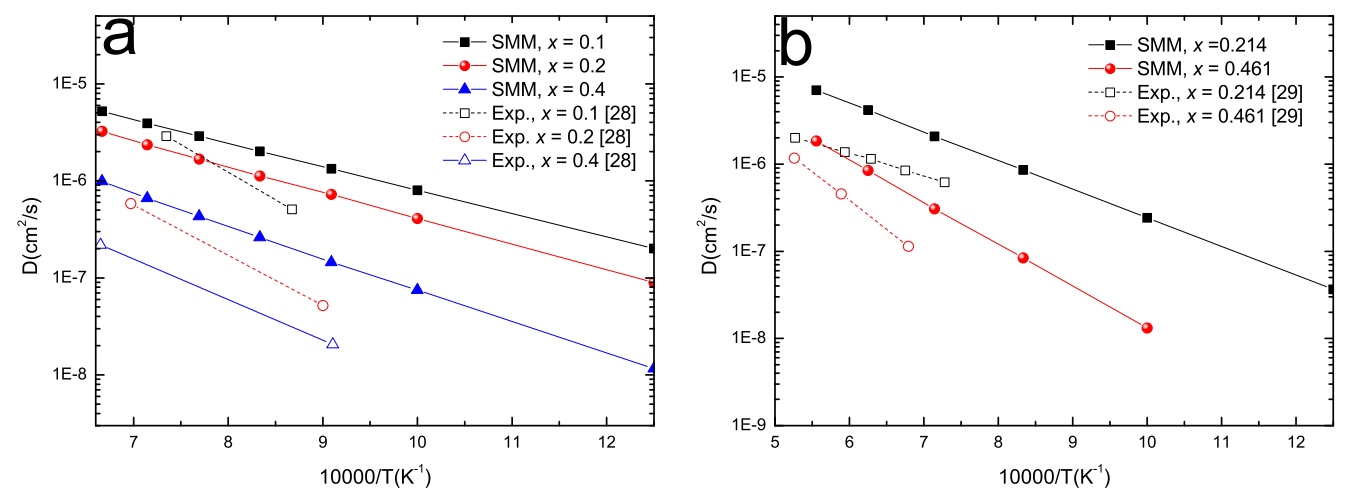

Fig. 4. The Arrhenius plots of the diffusion coefficient $D$ as a function of reciprocal temperature (1/T) at the different dopant concentration for YDC (a) and YSZ (b). The experimental results measured at $x=0.1,0.2,0.4$ for YDC [28] and at $x=0.214,0.461$ for YSZ [29] are presented for comparison.

\section{CONCLUSION}

We have presented an analytic formulation to study the oxygen vacancy diffusion in YDC and YSZ with fluorite structure. The present formalism takes into account the anharmonicity effects of thermal lattice vibrations and it enables us to derive the expression of vacancy activation 
energy in closed analytic forms. Our results show that the presence of dopant ions in the cation edges restricts the oxygen vacancy migration, and the oxygen vacancy hopping across the $\mathrm{R}^{4+}$ - $\mathrm{R}^{4+}$ edge contributes predominantly to the diffusion process. Consequently, the vacancy activation energies increase with the increasing dopant concentration and lead to a decrease of the vacancy diffusion coefficients. Our findings are in good agreement with the other theoretical and experimental results.

\section{REFERENCES}

[1] F. Ramadhaniac, M.A. Hussaina, H. Mokhlisb, S. Hajimolanad, Renewable and Sustainable Energy Reviews 76 (2017) 460.

[2] K. Muthukkumaran, R. Bokalawela, T. Mathews, S. Selladurai, J. Mater. Sci. 42 (2007) 7461.

[3] V.G. Zavodinsky, Physics of the Solid State 46 (2004) 453.

[4] H. Yoshida, T. Inagaki, K. Miura, M. Inaba, Z. Ogumi, Solid State Ionics 160 (2003) 109.

[5] F. Ye, T. Mori, D.R. Ou, A.N. Cormack, Solid State Ionics 180 (2009) 1127.

[6] T. Mori, T. Kobayashi, Y. Wang, J. Drennan,T. Nishimura, J.G. Li, H. Kobayashi, J. Am. Ceram. Soc. 88 (2005) 1981.

[7] H. Yoshida, T. Inagaki, K. Miura, M. Inaba, Z. Ogumi, Solid State Ionics 160 (2003) 109.

[8] T. Arima, K. Fukuyo, K. Idemitsu, Y. Inagaki, Journal of Molecular Liquids 113 (2004) 67.

[9] F. Pietrucci, M. Bernasconi, A. Laio, M. Parrinello, Physical Review B 78 (2008) 094301.

[10] A. Kushima, B. Yildiz, J. Mater. Chem. 20 (2010) 4809.

[11] M. Nakayama, M. Martin, Phys. Chem. Chem. Phys. 11 (2009) 3241.

[12] S. Grieshammer, B.O.H. Grope, J. Koettgen, M. Martin, Phys. Chem. Chem. Phys. 16 (2014) 9974.

[13] R. Krishnamurthy, Y.-G. Yoon, D.J. Srolovitz, R. Car, J. Am. Ceram. Soc. 87 (2004) 1821.

[14] R. Pornprasertsuk, P. Ramanarayanan, C.B. Musgrave, F.B. Prinz, Journal of Applied Physics 98 (2005) 103513

[15] V.V. Hung, B.D. Tinh, Modern Physics Letters B 25 (2011) 1101.

[16] K. Masuda-Jindo, V.V. Hung, P.E.A Turchi, Solid State Phenomena 138 (2008) 209.

[17] W. Chen, T.A. Lee, A. Navrotsky, J. Mater. Res. 20 (2005) 144.

[18] A. Bogicevic, C. Wolverton, Europhys. Lett. 56 (2001) 393.

[19] Z.-P. Li, T. Mori, J. Zou, J. Drennan, Materials Research Bulletin 48 (2013) 807.

[20] M. S. Khan, M. S. Islam, D.R. Bates, J. Mater. Chem. 8 (1998) 2299.

[21] R. Devanathan, W.J. Weber, S.C. Singhal, J.D. Gale, Solid State Ionics 177 (2006) 1251.

[22] P. Demontis, S. Spanu, G.B. Suffritti, J. Chem. Phys. 114 (2001) 7980.

[23] Z.-P. Li, T. Mori, F. Ye, D. Ou, G. J. Auchterlonie, J. Zou, J. Drennan, J. Phys. Chem. C 116 (2012) 5435.

[24] P.K. Schelling, S.R. Phillpot, J. Am. Ceram. Soc. 84 (2001) 2997.

[25] W. Zajac, J. Molenda, Solid State Ionics 179 (2008) 154.

[26] D.R. Ou, T. Mori, F. Ye, M. Takahashi, J. Zou, J. Drennan, Acta Materialia 54 (2006) 3737.

[27] S.P.S. Badwal, Solid State Ionics 52 (1992) 32.

[28] M.J.D. Rushton, A. Chroneos, S.J. Skinner, J.A. Kilner, R.W. Grimes, Solid State Ionics 230 (2013) 37.

[29] Y. Oishi, K. Ando, Transport in Nonstoichiometric Compounds 129 (1985) 189.

\section{APPENDIX}

The change in the average potential energy of an $\mathrm{O}^{2-}$ ion for the interactions between it and other $\mathrm{O}^{2-}$ ions due to the oxygen hopping to the saddle point

To determine $\Delta u_{O}^{O-O}$ in Eq. (26), we assume that when the $\mathrm{O}^{2-}$ ion is at the site A, the oxygen vacancy at the site $\mathrm{C}$ (Fig. 5) is occupied by an $\mathrm{O}^{2-}$ ion from the crystal lattice outside by some way. Consequently, the system has $\left(N_{O}+1\right) \mathrm{O}^{2-}$ ions, $\left(N_{v a}-1\right)$ oxygen vacancies and the total potential energy of these $\mathrm{O}^{2-}$ ions for the interactions between them and the other $\mathrm{O}^{2-}$ ions 
is $U_{O}^{*}$. Analogously, the $\mathrm{O}^{2-}$ ions at the sites $\mathrm{A}$ and $\mathrm{C}$ are vanished by some way. Then the total potential energy $U_{O}^{*}$ will be reduced by $\Delta u_{O}^{1}$, with $\Delta u_{O}^{1}$ being the total potential energy of the $\mathrm{O}^{2-}$ ions at the sites $\mathrm{A}$ and $\mathrm{C}$ for the reciprocal interactions between them and surrounding $\mathrm{O}^{2-}$ ions. In fact, the oxygen ion hops from the site $\mathrm{A}$ to the site $\mathrm{B}$ leading to the appearance of two oxygen vacancies at the sites $\mathrm{A}$ and $\mathrm{C}$ (Fig. 5).

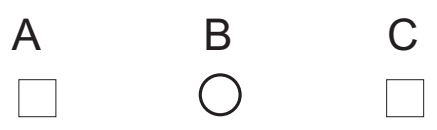

Fig. 5. The $\mathrm{O}^{2-}$ ion at the saddle point $\mathrm{B}$, and forming two oxygen vacancies at the lattice sites $\mathrm{A}$ and $\mathrm{C}$.

If one adds an oxygen ion to the site $\mathrm{B}$, the total interaction potential of the system is supplemented by $\Delta u_{O}^{2}$, with $\Delta u_{O}^{2}$ being the reciprocal interaction potential between the $\mathrm{O}^{2-}$ ion at the site $\mathrm{B}$ and surrounding $\mathrm{O}^{2-}$ ions. Therefore, the expression of $\Delta u_{O}^{O-O}$ is given by

$$
\Delta u_{O}^{O-O}=\frac{U_{O}^{*}-\Delta u_{O}^{1}+\Delta u_{O}^{2}}{N_{O}} .
$$

